\title{
The relationship between clinico-biochemical features in women with polycystic ovary syndrome and fertility treatment outcomes
}

\author{
Pedro Marques ${ }^{1}$, Florbela Ferreira ${ }^{2}$, Ana Paula Soares ${ }^{3}$, Joaquim Nunes ${ }^{3}$, Sandra Sousa ${ }^{3}$, Ana Aguiar $^{3}$, Carlos Calhaz-Jorge ${ }^{3}$ \\ 1Department of Endocrinology, Instituto Português de Oncologia de Lisboa, Portugal \\ 2Department of Endocrinology, Hospital Santa Maria, Lisboa, Portugal \\ ${ }^{3}$ Department of Obstetrics and Gynecology, Reproductive Medicine Unit, Hospital Santa Maria, Lisboa, Portugal \\ E-mail: pedro.miguel.sousa.marques@gmail.com
}

\section{Introduction}

- Polycystic ovary syndrome (PCOS) affects 5-8\% of reproductive-age women. The morbidity related to PCOS may include insulin-resistance, type 2 diabetes mellitus (T2DM), obesity, hypertension, cardiovascular disease and infertility.

- We aimed to evaluate the clinico-biochemical characteristics of PCOS infertile women and establish their relationship with fertility treatment outcomes.

\section{Methods}

- We reviewed the records of 229 PCOS women (Rotterdam criteria, 2003) surveilled at Hospital Santa Maria, between January 2004 and June 2013.

- Excluded cases of hyperprolactinemia; congenital adrenal hyperplasia; premature ovarian failure; hypogonadism hypogonadotropic.

- Fertility treatment outcomes were measured according to: 1) number of treatment cycles; 2) effective duration of the treatments.

\section{Results}

\begin{tabular}{|c|c|}
\hline Baseline features & $P C O S n=229$ \\
\hline Mean age $(y)$ & $29.7( \pm 3.9)$ \\
\hline Mean infertility duration (months) & $41( \pm 29)$ \\
\hline Primary infertility & $185(80.8 \%)$ \\
\hline Existence another infertility factor & $38(16.6 \%)$ \\
\hline Mean BMI $\left(\mathrm{kg} / \mathrm{m}^{2}\right)$ & $27.8( \pm 6.4)$ \\
\hline Weight excess (BMI $\geq 25 \mathrm{~kg} / \mathrm{m}^{2}$ ) & $134(58.5 \%)$ \\
\hline Mean waist circumference $(\mathrm{cm})$ & $93.6( \pm 14.5)$ \\
\hline Waist circumference $>80 \mathrm{~cm}$ & $169(73.8 \%)$ \\
\hline Hypertension & $12(5.2 \%)$ \\
\hline Familial history of T2DM & $72(31.4 \%)$ \\
\hline Smoking habits & $61(26.6 \%)$ \\
\hline Ovarian polycystic morphology & $213(93.0 \%)$ \\
\hline $\begin{array}{l}\text { Clinical and/or biochemical } \\
\text { androgen excess }\end{array}$ & $110(48.0 \%)$ \\
\hline Oligoamenorrea & $229(100 \%)$ \\
\hline Means: $\quad$ FSH $(2.5-10.2 \mathrm{U} / \mathrm{L})$ & $5.0( \pm 2.1)$ \\
\hline LH $(1.9-12.5 \mathrm{U} / \mathrm{L})$ & $9.0( \pm 5.7)$ \\
\hline Estradiol $(19.5-144.0 \mathrm{pg} / \mathrm{mL})$ & $67.9( \pm 65.0)$ \\
\hline Total testosterone $(<73 \mathrm{ng} / \mathrm{dL})$ & $63.6( \pm 33.0)$ \\
\hline Prolactin $(2.8-29.0 \mathrm{ng} / \mathrm{mL})$ & $12.5( \pm 6.3)$ \\
\hline TSH $(0.55-4.78 \mu \mathrm{U} / \mathrm{mL})$ & $2.6( \pm 1.9)$ \\
\hline 17-OH-Progesterone (0.1-3.0ng/mL) & $1.5( \pm 0.7)$ \\
\hline SHBG $(18-144 \mathrm{nmol} / \mathrm{L})$ & $43.3( \pm 38.1)$ \\
\hline Insulin (3-25 $\mathrm{mU} / \mathrm{L})$ & $12.1( \pm 9.6)$ \\
\hline Total cholesterol (<190 mg/dL) & $179.7( \pm 33.3)$ \\
\hline $\begin{array}{c}\text { Cholesterol-LDL }(<110 \mathrm{mg} / \mathrm{dL}) \\
\text { Cholesterol-HDL } \quad(>50 \mathrm{mg} / \mathrm{dL})\end{array}$ & $\begin{array}{l}111.5( \pm 33.7) \\
53.4( \pm 15.1)\end{array}$ \\
\hline Triglyceridemia (<150 mg/dL) & $86.8( \pm 42.9)$ \\
\hline Fasting glycemia (<110 mg/dL) & $96.1( \pm 27.7)$ \\
\hline Hypertriglyceridemia (>150 mg/dL) & $15(6.6 \%)$ \\
\hline Low cholesterol-HDL (<50 mg/dL) & $93(40.6 \%)$ \\
\hline Positive Oral Glucose Tolerance Test & $\begin{array}{l}23(10.1 \%) \\
{[\text { T2DM: } n=2]}\end{array}$ \\
\hline Hypothyroidism (TSH>4.78 $\mu \mathrm{U} / \mathrm{mL}$ ) & $17(7.4 \%)$ \\
\hline
\end{tabular}

Table 1: Baseline clinico-biochemical features of PCOS

\begin{tabular}{|cc|}
\hline Evolutive pregnancy achievement rate & $71.6 \%(\mathrm{n}=164)$ \\
\hline Number of women who had spontaneous abortions (SA) & $\mathrm{n}=34(14.9 \%)$ \\
\hline Total number of SA ( $\mathrm{n})$ & $43[5$ women had $>1]$ \\
\hline Mean treatment cycles to achieve evolutive pregnancy & $2.8( \pm 2.4)$ \\
\hline Mean duration of effective treatment (months) & $4.4( \pm 6.0)$ \\
\hline Mean duration between 1 st treatment and evolutive pregnancy (months) & $9.9( \pm 10.8)$ \\
\hline $\begin{array}{c}\text { Method of achievement pregnancy (n): } \\
\text { CC / Gnd / CC+Gnd / Spnt / OD / IUI/ FIV or ICSI }\end{array}$ & $25 / 60 / 15 / 16 / 30 / 12 / 6$ \\
\hline
\end{tabular}

Table 2: Overview of the results from fertility treatment in the 229 PCOS women cohort CC, clomiphene citrate; Gnd, gonadotropins; spnt, spontaneous; OD, ovarian drilling; IUI, intrauterine insemination; FIV, fertilization in vitro; ICSI, intracytoplasmic sperm insemination.

\begin{tabular}{|c|c|c|c|}
\hline \multicolumn{2}{|c|}{$\begin{array}{l}\text { PCOS women that obtained } \\
\text { evolutive pregnancy }(n=164)\end{array}$} & \multirow{2}{*}{$\begin{array}{l}\begin{array}{c}\text { Number of } \\
\text { treatment } \\
\text { cycles }(n)\end{array} \\
2.6( \pm 2.3) \\
2.6( \pm 1.9)\end{array}$} & \multirow{2}{*}{\begin{tabular}{|c|}
$\begin{array}{c}\text { Effective duration } \\
\text { of treatment } \\
\text { (months) }\end{array}$ \\
$4.05( \pm 5.3)$ \\
$2.93( \pm 2.4)$
\end{tabular}} \\
\hline $\begin{array}{l}\text { Age }<35 \text { years } \\
\text { Age }>35 \text { years }\end{array}$ & $\begin{array}{c}150 \\
14\end{array}$ & & \\
\hline $\begin{array}{l}\text { Primary infertility } \\
\text { Secondary infertility }\end{array}$ & $\begin{array}{c}132 \\
32\end{array}$ & $\begin{array}{l}2.9( \pm 2.3) \\
1.8( \pm 1.4)\end{array}$ & $\begin{array}{l}4.4( \pm 5.5) \\
2.3( \pm 2.3)\end{array}$ \\
\hline $\begin{array}{c}\text { Clinical and/or biochemical } \\
\text { androgen excess }\end{array}$ & $\begin{array}{l}\text { Yes } 82 \\
\text { No } 82\end{array}$ & $\begin{array}{l}2.9( \pm 2.5) \\
2.3( \pm 1.8)\end{array}$ & $\begin{array}{l}4.1( \pm 4.4) \\
3.9( \pm 5.8)\end{array}$ \\
\hline $\begin{array}{l}\text { Weight excess } \\
\left(B M I \geq 25 \mathrm{~kg} / \mathrm{m}^{2}\right)\end{array}$ & $\begin{array}{l}\text { Yes } 98 \\
\text { No } 66\end{array}$ & $\begin{array}{l}2.7( \pm 2.2) \\
2.5( \pm 2.3)\end{array}$ & $\begin{array}{l}3.9( \pm 3.4) \\
4.1( \pm 2.8)\end{array}$ \\
\hline $\begin{array}{l}\text { Waist circumference } \\
>80 \mathrm{~cm}\end{array}$ & $\begin{array}{l}\text { Yes } \mathbf{1 2 6} \\
\text { No } \mathbf{3 8}\end{array}$ & $\begin{array}{l}2.6( \pm 2.1) \\
2.3( \pm 1.8)\end{array}$ & $\begin{array}{l}3.8( \pm 3.8) \\
4.1( \pm 2.1)\end{array}$ \\
\hline Hypertension & $\begin{array}{c}\text { Yes } 7 \\
\text { No } 157\end{array}$ & $\begin{array}{l}2.1( \pm 0.9) \\
2.7( \pm 2.3)\end{array}$ & $\begin{array}{l}3.1( \pm 3.1) \\
3.9( \pm 5.2)\end{array}$ \\
\hline Familial history of T2DM & $\begin{array}{l}\text { Yes } \mathbf{5 3} \\
\text { No } \mathbf{1 1 1} \\
\end{array}$ & $\begin{array}{l}3.4( \pm 2.5) \\
2.3( \pm 2.0)\end{array}$ & $\begin{array}{l}4.2( \pm 3.6) \\
3.9( \pm 5.7)\end{array}$ \\
\hline Smoking habits & $\begin{array}{l}\text { Yes } 43 \\
\text { No } 121\end{array}$ & $\begin{array}{l}2.6( \pm 1.9) \\
2.7( \pm 2.3)\end{array}$ & $\begin{array}{l}3.8( \pm 4.0) \\
4.0( \pm 5.4)\end{array}$ \\
\hline $\begin{array}{l}\text { Hypertriglyceridemia } \\
(>150 \mathrm{mg} / \mathrm{dL})\end{array}$ & 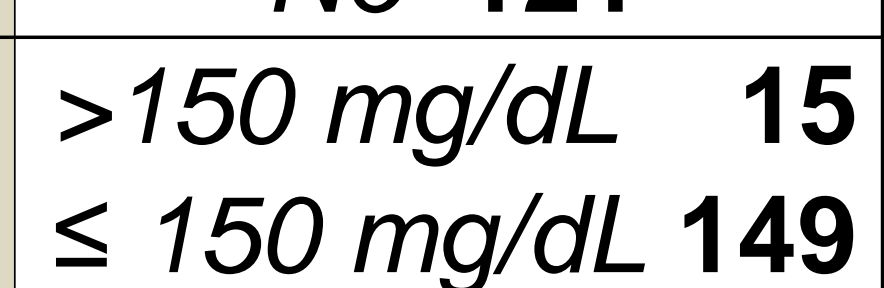 & $\begin{array}{l}3.6( \pm 2.9) \\
2.4( \pm 1.8)\end{array}$ & $\begin{array}{l}4.5( \pm 3.2) \\
3.4( \pm 3.8)\end{array}$ \\
\hline $\begin{array}{l}\text { Low cholesterol-HDL } \\
(<50 \mathrm{mg} / \mathrm{dL})\end{array}$ & $\begin{array}{l}<50 \mathrm{mg} / \mathrm{dL} 77 \\
\geq 50 \mathrm{mg} / \mathrm{dL} 87\end{array}$ & $\begin{array}{l}2.9( \pm 2.1) \\
2.2( \pm 1.8)\end{array}$ & $\begin{array}{l}3.5( \pm 3.6) \\
3.4( \pm 3.9)\end{array}$ \\
\hline $\begin{array}{l}\text { Oral Glucose } \\
\text { Tolerance Test }\end{array}$ & $\begin{array}{c}\text { Positive } 22 \\
\text { Negative } 142\end{array}$ & $\begin{array}{l}2.5( \pm 2.3) \\
2.6( \pm 1.9)\end{array}$ & $\begin{array}{l}3.43( \pm 3.9) \\
3.6( \pm 3.8)\end{array}$ \\
\hline $\begin{array}{c}\text { Hypothyroidism } \\
\text { (TSH }>4.78 \mu \mathrm{U} / \mathrm{mL})\end{array}$ & $\begin{array}{l}\text { Yes } 14 \\
\text { No } 150\end{array}$ & $\begin{array}{l}3.6( \pm 1.9) \\
2.6( \pm 2.2)\end{array}$ & $\begin{array}{c}5.2( \pm 4.6) \\
3.84( \pm 5.2)\end{array}$ \\
\hline
\end{tabular}

Table 3: Number and effective duration of treatment in the cohort of 164 PCOS that obtained pregnancy Yellow cells are indicative of differences with statistical significance $(p<0.05)$. Pearson correlation analysis between all biochemical parameters and the fertility outcomes have been done and no significant correlations were obtained

\section{Discussion}

Infertile PCOS women with primary infertility, T2DM familial history, hypertriglyceridemia and low cholesterol-HDL may have poorer fertility treatment results, possibly justifying more intensive approach. Other clinico-biochemical features seem not to have prognostic value for fertility treatments. 